FedUni ResearchOnline https://researchonline.federation.edu.au

This is the peer-reviewed version of the following article:

Abu-Siada, A., Islam, S. (2013) Image processing-based on-line technique to detect power transformer winding faults. 39th Annual Conference of the IEEE Industrial Electronics Society, IECON 2013; Vienna, Austria; 10th-14th November 2013 p. 1-6.

Which has been published in final form at: https://doi.org/10.1109/IECON.2013.6700042

Copyright (C 2013 IEEE. Personal use of this material is permitted. Permission from IEEE must be obtained for all other uses, in any current or future media, including reprinting/republishing this material for advertising or promotional purposes, creating new collective works, for resale or redistribution to servers or lists, or reuse of any copyrighted component of this work in other works. 


\title{
Image Processing-Based On-Line Technique to detect Power Transformer Winding Faults
}

\author{
A. Abu-Siada, Senior Member, IEEE, S. Islam, Senior Member, IEEE \\ Electrical and Computer Engineering Department, Curtin University, Australia
}

\begin{abstract}
Frequency Response Analysis (FRA) has been growing in popularity in recent times as a tool to detect mechanical deformation within power transformers. To conduct the test, the transformer has to be taken out of service which may cause interruption to the electricity grid. Moreover, because FRA relies on graphical analysis, it calls for an expert person to analyse the results as so far, there is no standard code for FRA interpretation worldwide. In this paper an online technique is introduced to detect the internal faults within a power transformer by constructing the voltage-current $(V-I)$ locus diagram to provide a current state of the transformer health condition. The technique does not call for any special equipment as it uses the existing metering devices attached to any power transformer to monitor the input voltage, output voltage and the input current at the power frequency and hence online monitoring can be realised. Various types of faults have been simulated to assess its impact on the proposed locus. A Matlab code based on digital image processing is developed to calculate any deviation of the $V-I$ locus with respect to the reference one and to identify the type of fault.
\end{abstract}

Index Terms - Condition monitoring, Power transformer, Frequency response analysis, Digital image processing

\section{INTRODUCTION}

$\mathrm{I}_{\mathrm{p}}^{\mathrm{N}}$ $\mathrm{N}$ modern electrical transmission and distribution systems, power transformers are critical components within the network. In the event that a failure occurs in service, the impact can be far reaching. Not only causing extended outages, but costly repairs and potentially serious injury or fatality can result. The ageing transformer population increases the likelihood of failure so a reliable diagnostic tool is required to determine the current state and the remaining life of these assets. The majority of transformers currently in service were installed prior to 1980 and as a result the bulk of the population is approaching or has already exceeded its design life [1]. This poses a significant risk for utilities and other power network stakeholders as the impact of an in service transformer failure can be catastrophic. Transformer age is determined by the condition of its insulation and an ageing transformer population greatly increases the likelihood of failure. The mechanical forces that a transformer is exposed to during faults, switching transients and other disturbance events result in magnetic forces being imposed on the windings. If these forces exceed the withstand capability of the transformer, winding deformation can occur.
One of the reasons a transformer experiences mechanical damage to its windings is the loss of clamping pressure due to insulation degradation caused by ageing. With only minor winding damage the transformer is still capable of normal operation however its ability to withstand faults is greatly reduced. As a result of the ageing asset population it is becoming more critical to detect even slight winding deformations as early as possible. Transformers are expected to survive a number of short circuit faults without failure but once any significant winding deformation is produced, the likelihood of surviving further short circuits is greatly reduced because of the locally increased electromagnetic stresses. Furthermore, any reduction in winding clamping due to insulation shrinkage caused by ageing will also increase the probability of failure by reducing the mechanical strength of the winding assemblies [2]. Winding deformation can take many forms including radial buckling, conductor tilting, spiral tightening and collapse of the winding end supports. It is difficult to detect these types of internal fault with traditional testing techniques [3]. Frequency response analysis (FRA) is a powerful diagnostic method in detecting winding deformation, core and clamping structure for power transformers [4]. Since transformer windings can be modelled as a network of capacitance, resistance, self and mutual inductances; the values of these parameters are altered when a fault occurs on the winding, and hence the frequency response of the winding will change accordingly. The test is conducted by applying a sweep frequency voltage of low amplitude to a transformer terminal and measure the response voltage across the other terminal of the winding with reference to the tank [5]. While the testing method is relatively simple since the development of specific FRA test equipment, the interpretation of results remains a highly specialised area and requires expert personnel to determine the type and possible location of the fault [6]. The main drawback of FRA is that the transformer has to be switched off and taken out of service which may cause interruption to the power network. To prevent network interruption due to transformer outage for testing and to reduce the need for specialist analysis, an alternative on-line method to detect internal mechanical faults inside the transformer is required. This paper introduces a new on line technique to detect internal faults within a power transformer. The technique is relying on constructing a locus between the voltage difference in the high and the low voltage sides of a particular phase against the input current. A digital image processing technique is used to compare the healthy and faulty loci and to determine the type of fault. Unlike FRA which uses a sweep frequency in the range of $2 \mathrm{MHz}$, this technique is 
performed at the power frequency and hence online monitoring of transformer mechanical condition can be realised. Moreover, the method does not call for any additional equipment as it uses the metering equipment attached to any power transformer to monitor the input/output voltages and the input current. Also, the method does not call for an expert person to conduct or to analyse the results.

\section{PROPOSED TECHNIQUE}

The proposed technique is relying on constructing a locus diagram relating the transformer input current on the $\mathrm{x}$-axis and the difference between the input and output voltages of a particular phase on the y-axis. The relationship relating the above parameters can be derived using the single phase transformer equivalent circuit and its phasor diagram shown in Fig. 1.

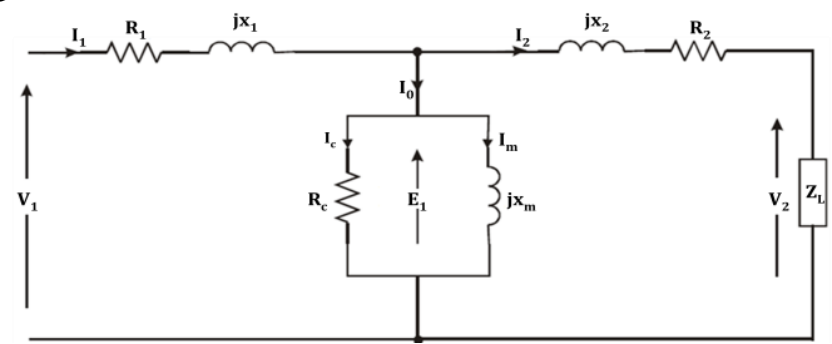

(a)

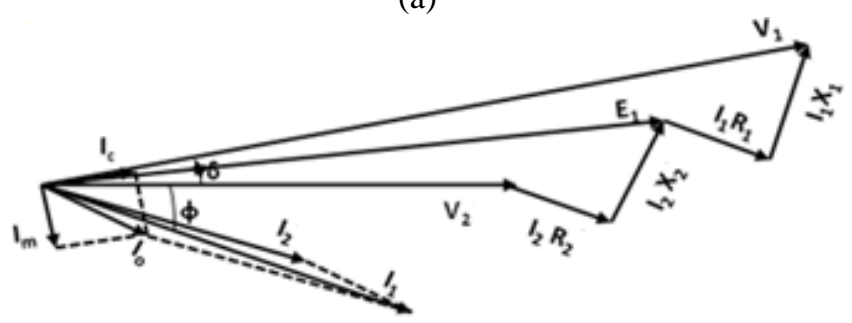

(b)

Fig. 1. (a) Per-unit equivalent circuit of transformer and (b) Phasor diagram

Let:

$\mathrm{v}_{2}(\mathrm{t})=V_{m 2} \sin (\omega \mathrm{t})$

$\mathrm{v}_{1}(\mathrm{t})=V_{m 1} \sin (\omega \mathrm{t}+\delta)$

$\mathrm{x}=\mathrm{i}_{1}(\mathrm{t})=I_{m 1} \sin (\omega \mathrm{t}-\varphi)$

For simplicity, assume $V_{m l}=V_{m 2}=V_{m}$

$\mathrm{y}=\mathrm{v}_{1}-\mathrm{v}_{2}=V_{m}\{\sin (\omega \mathrm{t}+\delta)-\sin (\omega \mathrm{t})\}$

$\Rightarrow \mathrm{y}=2 V_{m} \cos \left(\omega t+\frac{\delta}{2}\right) \cdot \sin \frac{\delta}{2}$

Cartesian formula relating $x$ and $y$ can be obtained from the parametric equations (3) and (5) by eliminating wt. From (3) and (5):

$$
\begin{aligned}
& \omega t=\sin ^{-1}\left(\frac{\mathrm{x}}{\mathrm{I}_{\mathrm{m} 1}}\right)+\varphi=\cos ^{-1}\left(\frac{\mathrm{y}}{2 \mathrm{~V}_{\mathrm{m}} \sin \frac{\delta}{2}}\right)-\frac{\delta}{2} \\
& \Rightarrow\left\{\cos ^{-1} \frac{y}{2 V_{m} \sin \frac{\delta}{2}}-\sin ^{-1} \frac{x}{I_{m 1}}\right\}=\left(\varphi+\frac{\delta}{2}\right)
\end{aligned}
$$

$$
\Rightarrow \sin \left\{\cos ^{-1} \frac{y}{2 V_{m} \sin \frac{\delta}{2}}-\sin ^{-1} \frac{x}{I_{m 1}}\right\}=\sin \left(\varphi+\frac{\delta}{2}\right)
$$$$
\Rightarrow \sin \left(\cos ^{-1} \frac{y}{2 V_{m} \sin \frac{\delta}{2}}\right) \cdot \cos \left(\sin ^{-1} \frac{x}{I_{m 1}}\right)-
$$$$
\cos \left(\cos ^{-1} \frac{y}{2 V_{m} \sin \frac{\delta}{2}}\right) \cdot \sin \left(\sin ^{-1} \frac{x}{I_{m 1}}\right)=\sin \left(\varphi+\frac{\delta}{2}\right)
$$$$
\Rightarrow \frac{\sqrt{\left(2 V_{m} \sin \frac{\delta}{2}\right)^{2}-y^{2}}}{2 V_{m} \sin \frac{\delta}{2}} \cdot \frac{\sqrt{I_{m}^{2}-x^{2}}}{I_{m 1}}-\frac{y}{2 V_{m} \sin \frac{\delta}{2}} \cdot \frac{x}{I_{m 1}}=
$$

$\sin \left(\varphi+\frac{\delta}{2}\right)$

$\Rightarrow \sqrt{\left\{\left(2 V_{m} \sin \frac{\delta}{2}\right)^{2}-y^{2}\right\} \cdot\left\{I_{m 1}^{2}-x^{2}\right\}}=$

$2 V_{m} I_{m 1} \sin \frac{\delta}{2} \sin \left(\varphi+\frac{\delta}{2}\right)+x y$

Squaring both sides and rearranging the equation:

$$
\begin{aligned}
& \left(2 V_{m} \sin \frac{\delta}{2}\right)^{2} x^{2}+4 V_{m} I_{m 1} \sin \frac{\delta}{2} \sin \left(\varphi+\frac{\delta}{2}\right) x y+I_{m 1}^{2} y^{2} \\
& +\left(2 V_{m} I_{m 1} \sin \frac{\delta}{2} \sin \left(\varphi+\frac{\delta}{2}\right)\right)^{2}-\left(2 V_{m} \mathrm{I}_{\mathrm{m} 1} \sin \frac{\delta}{2}\right)^{2}=0
\end{aligned}
$$

Assume the coefficients of (7) are:

$$
\begin{aligned}
& A=\left(2 V_{m} \sin \frac{\delta}{2}\right)^{2} ; B=4 V_{m} I_{m 1} \sin \frac{\delta}{2} \sin \left(\varphi+\frac{\delta}{2}\right) \\
& C=I_{m 1}^{2} ; D=\left(2 V_{m} I_{m 1} \sin \frac{\delta}{2} \sin \left(\varphi+\frac{\delta}{2}\right)\right)^{2}-\left(2 V_{m} \mathrm{I}_{\mathrm{m} 1} \sin \frac{\delta}{2}\right)^{2}
\end{aligned}
$$

The quadratic equation (7) represents [7]

$$
\begin{aligned}
& \text { a) an ellipse if } \mathrm{B}^{2}-4 A C \prec 0 \\
& \text { b) A parabola if } \mathrm{B}^{2}-4 A C=0 \\
& \text { c) A hyperbola if } \mathrm{B}^{2}-4 A C \succ 0 \\
& \mathrm{~B}^{2}-4 A C=16 I_{m 1}^{2} V_{m}^{2}\left\{\sin ^{2} \frac{\delta}{2} \sin ^{2}\left(\varphi+\frac{\delta}{2}\right)-\sin ^{2} \frac{\delta}{2}\right\} \\
& =16 I_{m 1}^{2} V_{m}^{2} \sin ^{2} \frac{\delta}{2}\left\{\sin ^{2}\left(\varphi+\frac{\delta}{2}\right)-1\right\} \\
& =-16 I_{m 1}^{2} V_{m}^{2} \sin ^{2} \frac{\delta}{2} \cdot \cos ^{2}\left(\varphi+\frac{\delta}{2}\right)
\end{aligned}
$$

Equation (8) is always negative term regardless the values of $I_{m}, V_{m}, \delta$ and $\varphi$. Hence the cartesian relationship between $\left(v_{1}-v_{2}\right)$ and $i_{1}$ represents an ellipse. As the phase shift between $V_{l}$ and $V_{2}$ is normally small, the impact of the angle $\delta$ on the locus is insignificant and can be neglected. The phase shift between $I_{1}$ and $V_{2}(\varphi)$ is almost equal to the load impedance phase angle as the phase shift between $I_{1}$ and $I_{2}$ is negligible. To investigate the impact of the load $\left(Z_{L}\right)$ power factor on the proposed locus, the $\Delta V-I_{I}$ locus is constructed for a $15-\mathrm{kVA}$, $2300 / 230 \mathrm{~V}$ single phase transformer with the following 
equivalent circuit parameters referred to the low voltage side:

$\mathrm{R}_{\mathrm{eq}}=4.45 \Omega ; \mathrm{X}_{\mathrm{eq}}=6.45 \Omega ; \mathrm{X}_{\mathrm{m}}=11 \mathrm{k} \Omega ; \mathrm{R}_{\mathrm{c}}=105 \mathrm{k} \Omega$.

Three operating conditions ( 0.8 lagging power factor, unity power factor and 0.8 leading power factor) with constant impedance magnitude are investigated and the corresponding $\Delta V-I_{I}$ locus for each case is constructed. The three loci are found to be identical as shown in Fig. 2. Hence, the load power factor has no impact on the proposed locus.

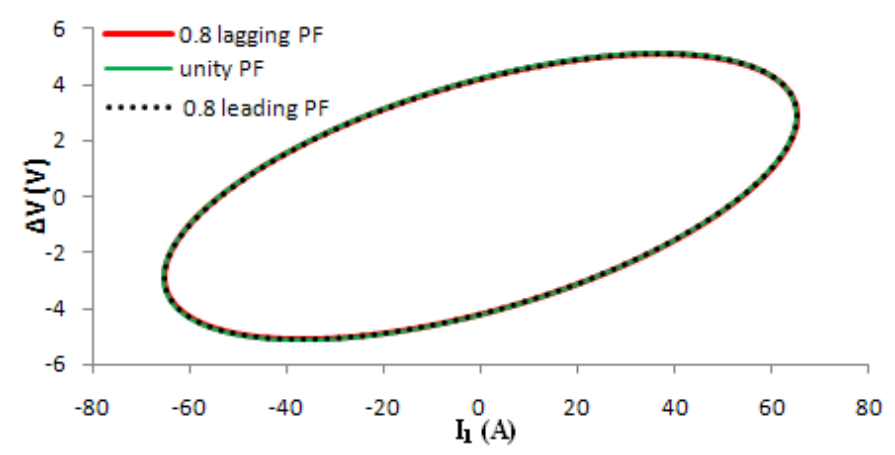

Fig. 2. Impact of load PF on the $\Delta V-I_{l}$ locus

\section{TRANSFORMER MODEL}

The transformer can be modeled with sufficient accuracy as a distributed analogue R-L-C circuit. Miki et al. [8] shows that the effect of the iron core had minimal role to play in an impulse stressed winding. This agrees well with the fact that in a rapid transient condition the flux lines tend to centre around the conductors rather than penetrating the iron core and for high frequency components of surges the iron core acts effectively as an earthed boundary [9]. Some studies [10, 11] have neglected the effect of distributed shunt conductance which is considered as a valid assumption for impulse voltage distribution analysis in the case of a faultless transformer, but may not be adequate in the case of fault diagnosis. Neglecting shunt conductance in the equivalent circuit will eliminate the study of leakage fault inside a transformer which could have been caused by several reasons such as insulation damage, ground shield or hot spots. The equivalent model (neglecting shunt conductance) could be ideal for verifying measured transfer function for inter-disk, coil short circuit and winding displacements. Hence the model needs some modifications to incorporate the study of leakage faults and partial discharges in the winding. These shortcomings of the computational model can be overcome if parameters which would allow for simulation of ground leakage and voids in the insulation are taken into consideration. The distributed transformer model equivalent circuit shown in Fig. 3 has been proposed in this paper.

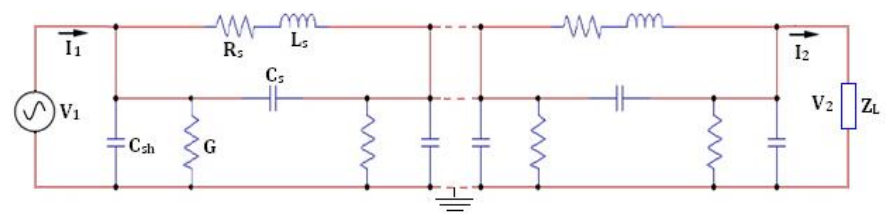

Fig. 3. Transformer distributed parameters model.

In this model, a single transformer winding is divided into cascaded pi-network comprising self/mutual inductances, resistance, series/shunt capacitances and shunt dielectric conductance. For simplicity, it is assumed that the mutual inductances are lumped into series inductances; this assumption is widely used in the literature [12]. The model parameters were calculated based on practical FRA measurements performed on the 3-phase low voltage windings of a 250 MVA, $345 / 16 \mathrm{kV}, 102$ disk transformer. Details of the model and parameter calculation based on FRA signature can be found in $[13,14]$. The use of distributed parameter model for power transformer will allow simulating different types of faults on the individual internal 102 disks used in this model. The parameters of the distributed model shown in Fig. 3 can also be determined from the geometrical dimensions of the transformer which makes the model suitable for different fault studies [12].

\section{SIMULATION RESULTS}

102 disks (two turns per disk) of the model shown in Fig. 3 is simulated using PSIM software. The model is energized by ac, $50 \mathrm{~Hz}$ voltage source of low amplitude and the instantaneous values of $v_{1}, v_{2}$ and $i_{1}$ are recorded at a time step of $10 \mu \mathrm{s}$. In this way a $\Delta V-I_{l}$ locus of a healthy transformer can be constructed and is considered as a reference or fingerprint of this transformer. When a transformer experiences an event that results in deformation of the windings, the transformer impedance will vary and this alters the transformer $\Delta V-I_{l}$ locus diagram.

As has been shown in Fig. 2, the load power factor with constant impedance magnitude does not have any impact on the proposed locus. Different load levels at constant power factor are simulated to investigate the effect of load magnitude variation on the proposed locus.

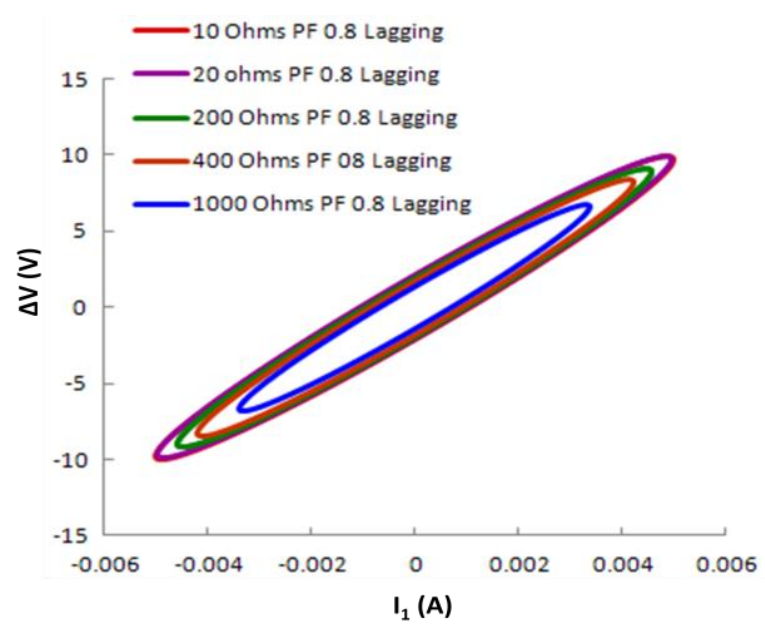

Fig. 4. Impact of load PF on the $\Delta V-I_{l}$ locus

As can be shown in Fig.4, increasing the load level from 10 $\Omega$ to $20 \Omega(100 \%$ increment) will not have any impact on the proposed locus. The effect of load magnitude on the proposed locus will take place when there is a significant change in load level as can be shown when the load magnitude is increased to $200 \Omega$. The effect of this significant increment in load magnitude on reducing the entire area of the $\Delta V-I_{I}$ locus is 
clearly shown in Fig. 4. However, all loci will have the same common major axis and same centroid. In the model under study, a $10 \angle 36.87^{\circ} \Omega$ is simulated as the load impedance.

Fig. 5 shows the $\Delta V-I_{1}$ locus of the healthy transformer as there was not any change made to the model parameters.

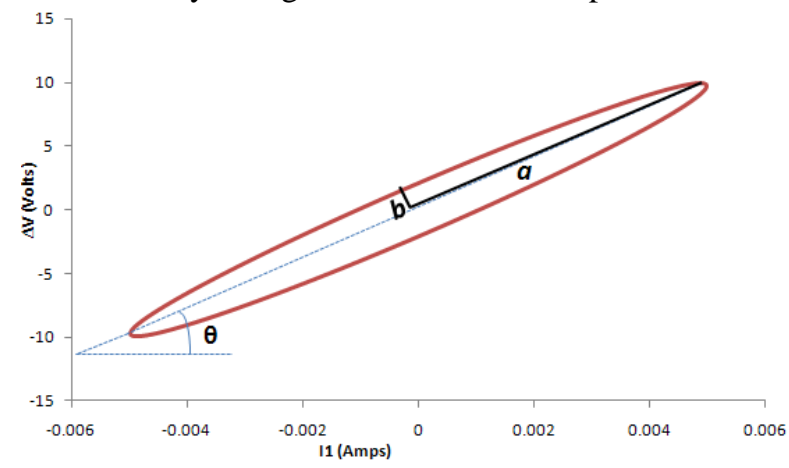

Fig.5. Healthy $\Delta V-I_{l}$ locus

Different mechanical faults are simulated on the model and the corresponding $\Delta V-I_{1}$ loci are plotted and compared with the healthy locus shown in Fig. 5 as will be discussed in the following sections. Diagnosis of the problem is achieved by comparing the healthy transformer fingerprint and the faulty one to identify any differences and hence to determine the possible fault type. In this paper, a Matlab code is developed to measure some unique features of the $\Delta V-I_{l}$ locus such as the semi-major and semi-minor axes lengths and the angle between the major axis and the horizontal axis. These parameters are shown in Fig. 5 as $a, b$ and $\theta$ respectively.

\section{FAULT ANALYSIS}

To identify the features of winding deformation and effect of model parameters on the $\Delta V-I_{l}$ locus, faults such as interdisk fault, axial displacement, and buckling stress of inner winding have been simulated and compared with the healthy locus.

\section{A. Inter-disk fault}

Inter-disk fault is considered as the most common fault of power transformer. Studies show that about $80 \%$ of transformer breakdowns are attributed to inter-disk fault [15, $16]$.

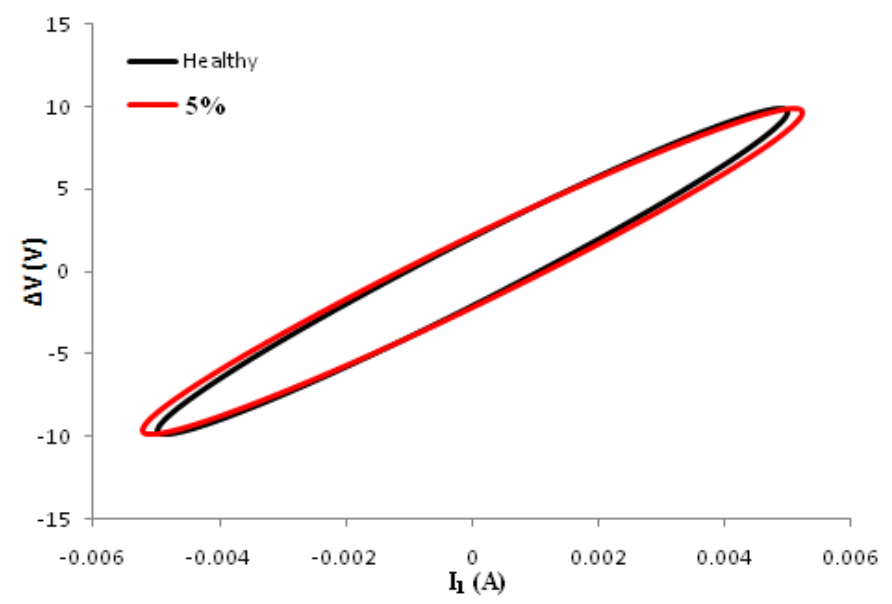

Fig. 6. Accuracy of the proposed approach to detect inter-disk fault
In the model under study, different number of disks has been short circuited to find its impact on the $\Delta V-I_{l}$ locus. To show the accuracy of the model to detect this fault, $5 \%$ of the coils have been short circuited and the proposed locus is compared to the healthy one as given in Fig. 6 which clearly shows the difference between the two loci.

Fig. 7 shows the locus for $30 \%$ and $60 \%$ faulty disks compared to the healthy locus. It can be observed from Fig. 6 and Fig. 7 that as the number of faulty disks increase, the locus rotates in clockwise direction and its entire area increases.

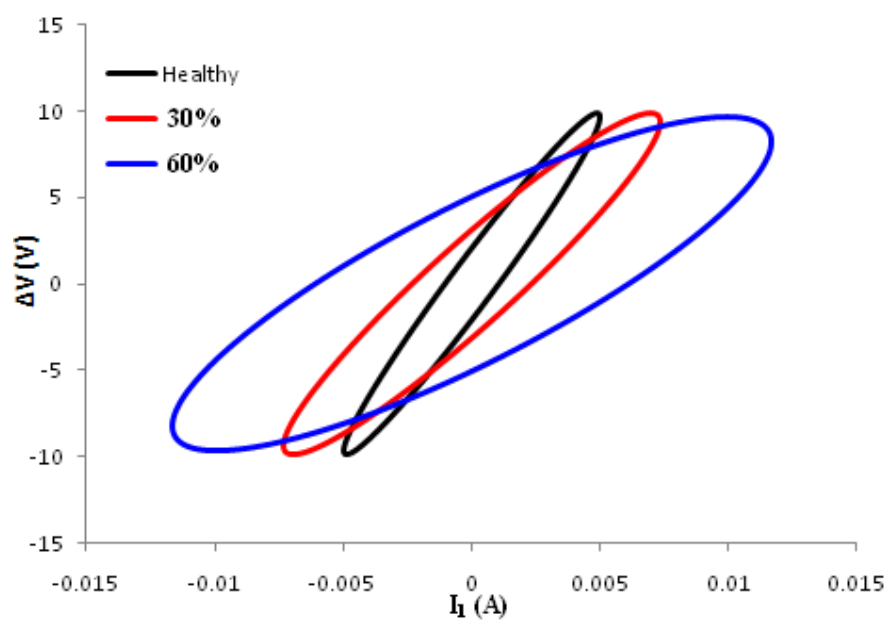

Fig. 7. Effect of inter-disk fault on $\Delta V-I_{I}$ locus

\section{B. Axial displacement}

This fault occurs due to the magnetic imbalance between the low and high voltage windings due to short circuit currents. The axial displacement between the magnetic centers of the windings will result in unbalanced magnetic force components in each half of the winding which leads to a change in its relative position. Leaving this fault unattended can cause winding collapse or failure of the end-supporting structure due to its progressive nature. This type of fault can be simulated by changing the mutual and self inductances of particular disks. Change in capacitance can be neglected [17]. In the model under study, axial displacement is modeled by $10 \%$ decrease in the inductance.

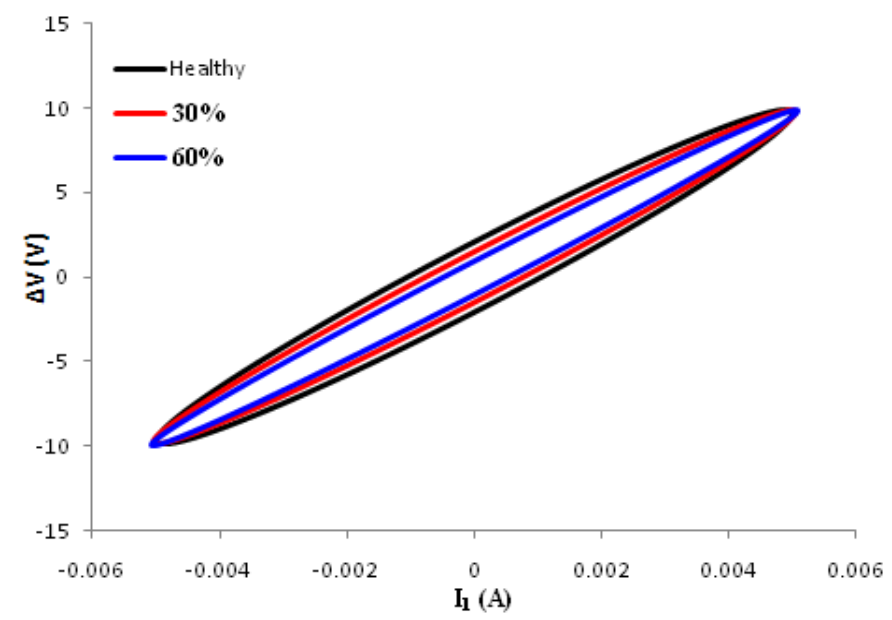

Fig.8. Effect of Axial displacement on $\Delta V-I_{I}$ locus 
Fig. 8 shows the effect of axial displacement of $30 \%$ and $60 \%$ disks on the $\Delta V-I_{1}$ locus. Fig. 8 shows that axial displacement will decrease the area of the faulty locus compared with the healthy one. Increasing the number of faulty disks will further decrease the locus area but there is no rotation in the locus major axis.

\section{Buckling stress}

Leakage flux and current in the windings causes radial force on windings. This force pulls the inner windings close to the core (buckling stress), while pushing the outer winding toward the limb (tensile stress) [18]. Buckling stress can be simulated in the distributed model by reducing the inter-winding capacitance and the mutual inductance between the windings at the position of deformation. Furthermore, the shunt capacitance is increased due to the reduction of the distance between winding and the core [19].

In this paper, forced buckling is modeled by increasing the shunt capacitance by $10 \%$, decreasing the inductance and series capacitance by $10 \%$. The effect of such fault on the proposed locus is shown in Fig. 9. Unlike axial displacement effect, buckling stress is increasing the locus area and the major axis will slightly rotate in the clockwise direction as the number of faulty disks increases. The slight locus rotation discriminates this type of fault from the inter-disk fault.

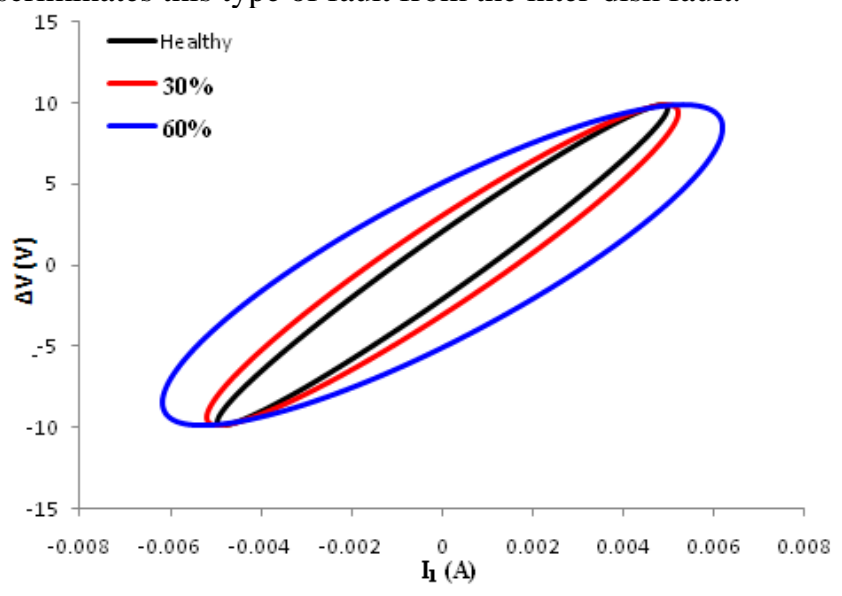

Fig. 9. Effect of forced buckling on $\Delta V-I_{I}$ locus

\section{IMAGE PROCESSING TECHNIQUE}

As has been shown in the mathematical proof and simulation results above, the $\Delta V-I_{l}$ locus is always representing an ellipse. Some unique features of ellipse can be used to compare different loci and to identify the type of fault within the power transformer. These features include ellipse centroid, the major and minor axes lengths ( $a$ and $b$ respectively), the angle between the major axis and the horizontal axis $(\theta)$. A Matlab code is developed to measure these parameters and to calculate the ellipse eccentricity which is used to describe the ellipse general proportion.

To identify the type of fault based on eccentricity, angle of rotation, major-axis length and minor- axis length, each fault has been simulated on different number of disks starting from 5 disks to 100 disks and these parameters are calculated for each fault using the developed software.

Results show that inter-disk fault has a significant increase in the eccentricity and angle of rotation as the number of faulty disks increases. Axial displacement does not introduce any effect on the axis rotation and the value of eccentricity is slightly increasing as the number of faulty disks increase. The eccentricity in forced buckling is slightly increasing with the increase of faulty disks. On the other hand, the increase in the angle of rotation with the increase of faulty disks is significant in case of forced buckling. Based on the range of the percentage differences of these parameters for each fault, the Matlab code is modified to identify the type of fault within the transformer. Two case studies are investigated as below.

Case 1: two identical loci are compared using the developed software. The developed software converts the colour of the two loci into white with black background to perform the calculations of ellipse centroide, major and minor axes lengths, eccentricity and the angle between the major axis and the horizontal axis. The software produces the two loci shown in Fig. 10 and shows that there is no difference in eccentricity and angle of rotation of the two loci and hence the software recommends a healthy transformer for this case.
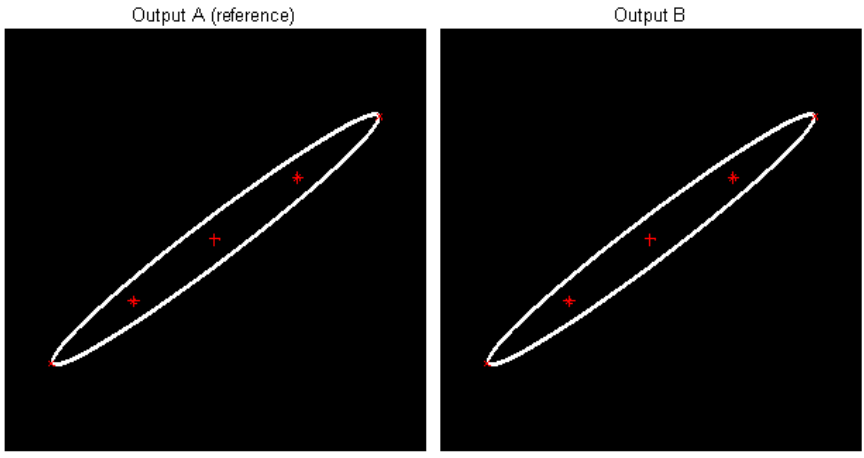

Fig. 10. Comparison of two identical loci

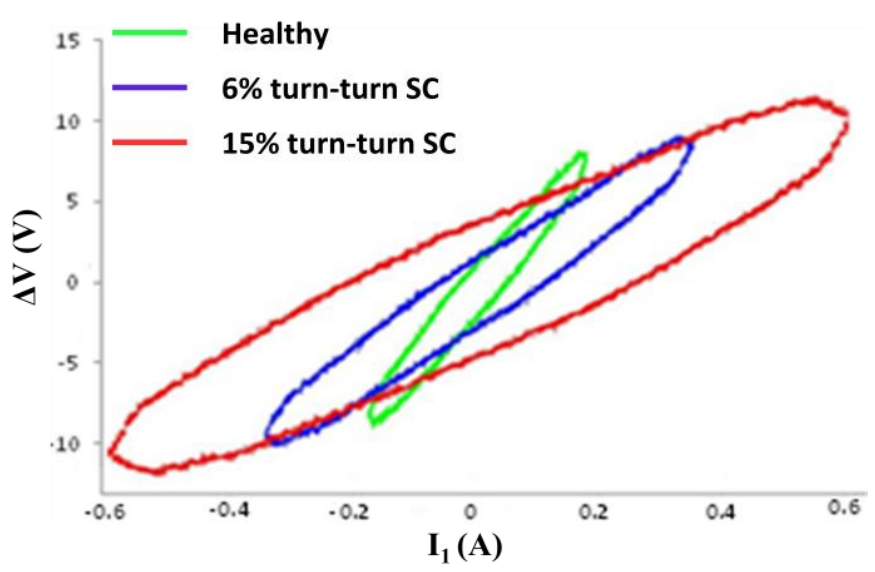

Fig. 11. Effect of practical Turn to Turn Faults on $\Delta V-I_{l}$ Locus

Case 2: A laboratory experimental testing was performed on a $0.5 \mathrm{kVA}, 150 / 170 \mathrm{~V}$ single phase transformer. The transformer is loaded by a $54 \Omega$ resistor and a turn-turn short 
circuit is created on $6 \%$ and $15 \%$ of the low voltage winding. The $\Delta V-I_{I}$ locus of the transformer is constructed using a digital oscilloscope. The healthy and faulty loci are compared as shown in Fig. 11 which shows a significant change in the locus area as the number of faulty turns increases. The healthy and $6 \%$ short circuit turns loci were fed to the developed software, the percentage difference in eccentricity calculated by the software is $0.21 \%$ and the percentage difference in the angle of rotation calculated by the software is $11.9 \%$; these differences are clearly visible in the two loci shown in Fig. 12 that are generated by the developed software.
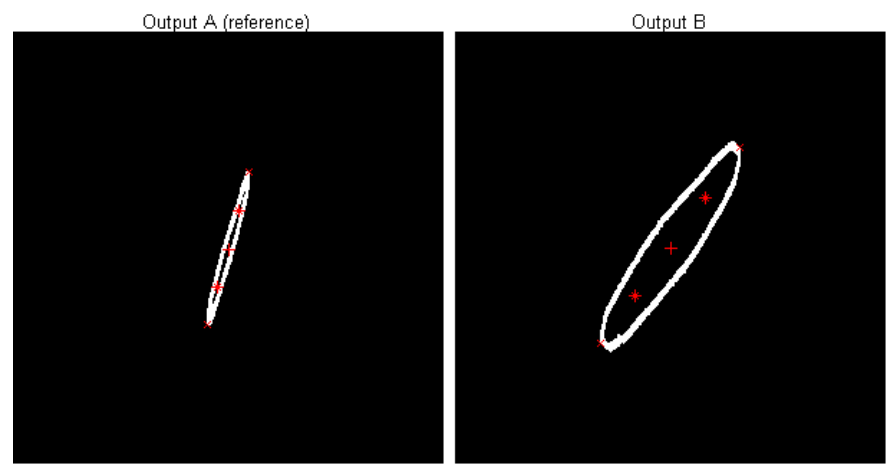

Fig. 12. Comparison of practical faulty and healthy loci

\section{CONCLUSIONS}

This paper presents a new technique to identify mechanical faults within a power transformer. The technique relies on constructing a locus diagram of the input and output voltage difference of a particular transformer winding on the $\mathrm{Y}$-axis and the winding input current on the X-axis. This locus is considered as the finger print of the transformer. Any mechanical fault will alter this locus in a unique way and hence fault detection as well as a fault type can be identified. Digital image processing technique based on measuring and comparing some features of the loci to identify the possible fault type is developed. These features include image centroid, the major and minor axes lengths, eccentricity and the angle of rotation. Simulation results show that each fault has a unique impact on these parameters. The axial displacement does not have any impact on the angle of rotation and has a minor impact on eccentricity. Inter-disk fault has significant impact on angle of rotation and eccentricity while forced buckling has moderate impact on the angle and its impact on the eccentricity is minor. The technique does not call for any new hardware as it uses the existing metering devices attached with the power transformer and can be implemented online as it is performed at the power frequency. The proposed locus can be plotted every cycle (20 ms based on $50 \mathrm{~Hz}$ network) and compared with the previous locus using the developed image processing code to immediately identify any changes and generates early warning signal.

\section{REFERENCES}

[1] E. J. Figueroa, "Managing an Aging Fleet of Transformers," in 6th Southern africa Regional conference, Cigre 2009, 2009.

[2] S. M. Islam and G. Ledwich, "Locating transformer faults through sensitivity analysis of high frequency modeling using transfer function approach," in Electrical Insulation, 1996., Conference Record of the 1996 IEEE International Symposium on, 1996, pp. 38-41 vol.1.

[3] M. S. A. Minhas, J. P. Reynders, and P. J. De Klerk, "Failures in power system transformers and appropriate monitoring techniques," in High Voltage Engineering, 1999. Eleventh International Symposium on (Conf. Publ. No. 467), 1999, pp. 94-97 vol.1.

[4] A. A. Reykherdt and V. Davydov, "Case studies of factors influencing frequency response analysis measurements and power transformer diagnostics," Electrical Insulation Magazine, IEEE, vol. 27, pp. 22-30.

[5] H. Firoozi, M. Kharezi, H. Rahimpour, and M. Shams, "Transformer Fault Diagnosis Using Frequency Response Analysis - Practical Studies," in Power and Energy Engineering Conference (APPEEC), 2011 AsiaPacific, pp. 1-4.

[6] "Mechanical-Condition Assessment of Transformer Windings Using Frequency Response Analysis (FRA)," CIGRE Working Group A2.26

2007.

[7] G. B. Thomas and R. L. Finney, Calculus and Analytic Geometry, 9 ed.: Addison-Wesley, 1996.

[8] A. Miki, T. Hosoya, and K. Okuyama, "A Calculation Method for Impulse Voltage Distribution and Transferred Voltage in Transformer Windings," power apparatus and systems, ieee transactions on, vol. PAS97, pp. 930-939, 1978.

[9] B. D. Sparling, "Transformer monitoring moving forward from monitoring to diagnostics," in Transmission and Distribution Conference and Exhibition 2002: Asia Pacific. IEEE/PES, 2002, pp. 1506-1509 vol.2.

[10]N. Abeywickrama, Y. V. Serdyuk, and S. M. Gubanski, "Effect of Core Magnetization on Frequency Response Analysis (FRA) of Power Transformers," Power Delivery, IEEE Transactions on, vol. 23, pp. 14321438, 2008.

[11]J. Pleite, C. Gonzalez, J. Vazquez, and A. Lazaro, "Power transformer core fault diagnosis using frequency response analysis," in Electrotechnical Conference, 2006. MELECON 2006. IEEE Mediterranean, 2006, pp. 1126-1129.

[12]Z. Chun, R. Jiangjun, D. Zhiye, L. Shoubao, Y. Yefeng, and Z. Yuanhang, "Calculation of parameters in transformer winding based on the model of multi-conductor transmission line," in Electrical Machines and Systems, 2008. ICEMS 2008. International Conference on, 2008, pp. 463-467.

[13]E. P. Dick and C. C. Erven, "Transformer Diagnostic Testing by Frequuency Response Analysis," Power Apparatus and Systems, IEEE Transactions on, vol. PAS-97, pp. 2144-2153, 1978.

[14] A. Abu Siada, S. Doglas and S. Islam, "Frequency Response Analysis using High Frequency Transformer Model", Presented at MEPCON, Egypt, December 2009.

[15]D. Sharafi, "Life Extension of a Group of Western Power Transformers," in Power and Energy Engineering Conference (APPEEC), 2010 AsiaPacific, pp. 1-4.

[16]S. M. Islam, "Detection of shorted turns and winding movements in large power transformers using frequency response analysis," in Power Engineering Society Winter Meeting, 2000. IEEE, 2000, pp. 2233-2238 vol.3.

[17]E. Rahimpour, J. Christian, K. Feser, and H. Mohseni, "Transfer Function Method to Diagnose Axial Displacement and Radial Deformation of Transformer Winding," Power Engineering Review, IEEE, vol. 22, pp. 70-70, 2002.

[18]E. Billig, "Mechanical stresses in transformer windings," Electrical Engineers - Part II: Power Engineering, Journal of the Institution of, vol. 93, pp. 227-243, 1946.

[19] J. A. S. B. Jayasinghe, Z. D. Wang, P. N. Jarman, and A. W. Darwin, "Winding movement in power transformers: a comparison of FRA measurement connection methods," Dielectrics and Electrical Insulation, IEEE Transactions on, vol. 13, pp. 1342-1349, 2006. 